\title{
QUINZE ANS D'OBSERVATIONS SUR LE GÈNE DE NANISME CHEZ LA POULE DOMESTIQUE
}

\author{
P. E. BERNIER ET G, H. ARSCOTT \\ Department of Poultry Science, \\ Oregon Agricultural Expériment Station, \\ Oregon State University, \\ Corvallis, Oregon, 9733I. (U.S.A.)
}

Les observations sur le nanisme à la S.E.A. de l'Oregon remontent à 1952, mais l'étude systématique n'a commencé qu'en 1956. Des mutants nains ont été observés cliez des Cornish et dans deux lignées consanguines de Leghorn et ont aussi été obtenus d'un accouveur local.

Une femelle mosaïque pour le nanisme et la taille normale a été observée dans la descendance de mâles nains accouplés à des femelles de taille normale. Cette femelle est génétiquement naine, toute la descendance l'étant. Un ergot s'est développé sur le tarse le plus grand. On suppose que le globule polaire contenant le chromosome sexuel porteur du gène pour la taille normale s'est réuni à l'une des deux cellules issues du premier clivage. Ceci a eu pour résultat une demi-femelle naine et un demi "neutre "triploïde de taille normale. L'analyse cytologique du sang et des follicules de plumes n'a pas révélé d'anomalie du caryotype.

Un létal récessif lié au sexe a été observé chez un mâle nain ayant une production de sperme exceptionnellement élevée. La moitié des embryons femelles issus du mâle porteur meurent très précocement et sont souvent classés comme infertiles. Des mâles nains accouplés à des femelles normales seraient un moyen commun d'obtenir des pondeuses naines, mais la disparité de taille pourrait éventuellement réduire le taux de fertilité dans les accouplements naturels.

Une proportion des mâles aux femelles de 1 à 15 et de 1 à 10 a donné jusqu'à cette année environ 80 p. 100 de fertilité. Une période d'au moins trois ou quatre semaines est apparue nécessaire pour atteindre ce niveau de fertilité, quoique celle-ci ait continué à augmenter lentement et pendant 30-35 jours. Dans la période 1966-1971, les mâles ont été transférés directement de locaux sombres à des parquets éclairés avec les femelles. Cette année, les mâles étaient d'abord exposés à quinze heures de lumière pendant un mois avant d'être placés avec les femelles. La fertilité a augmenté beaucoup plus vite, atteignant 90 p. 100 en 15 jours, 95 p. 100 en 21 jours, mais la proportion était encore 1 mâle/10 femelles.

Les naines de lignée pure gardées en cages individuelles et reproduites par insémination artificielle ont montré une fertilité et un taux d'éclosion comparable à celles obtenues avec les femelles de taille normale.

Dans notre sélection génétique pour la production d'œufs des poules mises en poulaillers sur la base des performances individuelles et familiales, les résultats ont été pendant un certain temps désappointants. Ceci provient apparemment de ce que des polygènes pour l'hypothyroïdisme étaient en ségrégation par suite de la consanguinité qu'accompagnait notre sélection. L'âge au premier œuf a augmenté jusqu'à la valeur de 32,5 semaines, mais il a ensuite décru graduellement jusqu'à 24,3 semaines à la génération actuelle, ce qui représente encore une maturité de 1,7 semaine plus tardive que dans notre lignée de production $O S U$.

La production des poules mises en poulailler a été assez décevante pendant un certain nombre d'années, mais elle a commencé à s'améliorer dans les trois dernières générations. Elle représente maintenant 83,4 p. 100 de celle observée dans la lignée "Production "à 40 semaines d'âge. La taille des œufs a augmenté graduellement. Le poids corporel n'a pas changé à cause de la restriction à laquelle il était soumis dans notre sélection. Sinon, il aurait très vraisemblablement changé, comme réponse liée à notre sélection pour la taille des œufs.

Des études de nutrition ont été conduites avec des populations d'effectif allant de 240 animaux les premières années à plus de 1200 pondeuses naines dans les années plus récentes, par groupes de 60. Nous avons mis en évidence le fait que les poulettes naines, même compte tenu 
de leur maturité sexuelle retardée, ont besoin d'une quantité d'aliments plus faible pour atteindre l'âge de la ponte. Les études de nutrition ont porté sur l'influence :

1. des taux de calcium,

2. du doublement du taux normal de vitamines et d'oligo-éléments,

3. du taux protéïque jusqu'à 21 p. 100 ,

4. de la supplémentation en méthionine, lysine et acide linoléïque.

Quoique la production d'œufs ait plafonné à partir du taux protéique de 15 p. 100, la taille des œufs a continué à augmenter jusqu'à 21 p. 100 . L'addition de 0,05 p. 100 de méthionine a augmenté la ponte et le poids des œufs significativement et à des niveaux comparables à ceux observés avec une ration à 21 p. 100 de protéines. Actuellement, un faible taux de protamone est donné aux pondeuses élevées avec ou sans supplément de protamone, mais ce taux a dû être réduit de moitié, jusqu'à 0,0167 p. 100 , car il abaissait la ponte.

Les études physiologiques ont inclus l'observation des hypophyses, thyroïdes, surrénales et ovaires, à la fois pour des femelles en croissance et adultes, ainsi qu'un test biologique d'homogénats hypophysaires sur des rates immatures hypophysectomisées. Quoique les thyroïdes des femelles naines soient apparues plus petites que celles des normales à 7-13 mois d'âge, le test biologique n'a pu prouver que les modifications thyroïdiennes chez les naines soient dues à des altérations de l'activité thyréotrope de leur hypophyse.

Le résultat du test biologique des hypophyses ne confirme pas l'hypothèse de l'accumulation d'hormone somatotrope dans les hypophyses d'oiseaux nains. L'observation de l'ACTH et de l'activité gonatrodope a été faite aussi sur les hypophyses naines.

\title{
EFFET DE GÈNES LIÉS AU SEXE SUR LA TAILLE CORPORELIL ETT LA REPRODUCTION
}

\author{
R. GEORGE JAAP \\ Ohio State University \\ Colombus. Ohio 432 Io (U.S.A.)
}

Deux gènes au moins, situés sur le chromosome sexuel, réduisent la vitesse de croissance et la taille arlulte des poules. L'un est le gène de nanisme lié au sexe bien connu $d w$.Nous employons le symbole $B$ pour un autre gène lié au sexe parce qu'on le trouve habituellement dans les races Bantam. Les données présentes indiquent que $B$ et $d w$ peuvent être allèles, l'ordre de dominance décroissant chez les mâles hétérozygotes étant + (normal), $B$ et $d w$. Quand le poids corporel des femelles normales $(+)$ est d'environ $2 \mathrm{~kg}$, les femelles $d w$ atteignent 65 à 70 p. 10 de ce poids et les femelles $B 90$ p. 100 au moins du poids adulte des femelles normales. Dans les populations de type broiler où le poids adulte des femelles normales approche $4 \mathrm{~kg}$ ou plus, les femelles $d w$ atteignent 80 p. 100 de ce poids normal, contre $65-70$ p. 100 chez le type "ponte " de plus petite taille.

Les femelles naines $d w$ sont habituellement identifiables non seulement par leur petite taille, mais aussi par leurs tarses courts. Par contraste, les femelles $B$ ne peuvent être phénotypiquement distinguées des femelles normales et sont identifiables seulement lorsque le chromosome sexuel portant $B$ contient un marqueur phénotypique visible tel que l'emplumement rapide ou lent, ou la couleur du duvet argentée ou dorée. Le locus $I d$ (inhibiteur de la mélanine dermique), qui est presque indépendant des loci $S K$, ne permet pas d'identifier l'effet de $B$. 